Proyecciones

Vol. 20, No 1, pp. 33-51, May 2001.

Universidad Católica del Norte

Antofagasta - Chile

\title{
NONLINEAR ELLIPTIC PROBLEMS \\ WITH RESONANCE AT THE TWO \\ FIRST EIGENVALUE : A VARIATIONAL APPROACH
}

M. MOUSSAOUI and M. MOUSSAOUI

University Mohamed I - Oujda, Morocco

\begin{abstract}
We study the nonlinear elliptic problems with Dirichlet boundary condition

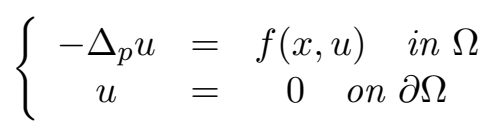

Resonance conditions at the first or at the second eigenvalue will be considered.
\end{abstract}

KEY WORDS : p-laplacian, eigenvalue, resonance, variational method. 


\section{INTRODUCTION}

Let us consider the Dirichlet problem

(1.1)where

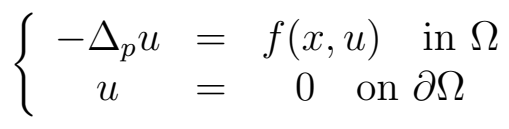

$\Omega$ is a bounded smooth domain in ${ }^{N}(N \geq 1)$ and the nonlinearity

$f: \Omega \times \rightarrow$ is assumed to be a Carathéodory function with subcritical growth, that is:

$$
|f(x, s)| \leq a|s|^{q-1}+b \quad \forall s \in ; \text { a.e. } x \in \Omega
$$

for some constants $a, b>0$, where $1 \leq q<p^{*}$, if $N>p$ and $1 \leq q<+\infty$ if $N \leq p$, with $\frac{1}{p^{*}}=\frac{1}{p}-\frac{1}{N}$.

$\Delta_{p}, 1<p<\infty$ is the p-laplacian $\Delta_{p} u=\operatorname{div}\left(|\nabla u|^{p-2} \nabla u\right)$. The operator $\Delta_{p}$ with $p \neq 2$ arises from a variety of physical phenomena. It is used in non-Newtonian fluids, in some reaction-diffusion problems as well as in flow through porous media. It appears also in nonlinear elasticity, glaceology and petroleum extraction. The linear case when $p=2$ has been studied by many authors, see e.g [13], [9], [6] $\cdots$

The nonlinear case $(p \neq 2)$, when the nonlinearity $\frac{p F(x, s)}{|s|^{p}}$ stays asymptotically between $\lambda_{1}$ and $\lambda_{2}$, where $F(x, s)$ denotes the primitive $F(x, s)=\int_{0}^{s} f(x, t) d t$ and $\lambda_{1}, \lambda_{2}$ are the first and the second eigenvalues of $-\Delta_{p}$ on $W_{0}^{1, p}(\Omega)$, has been studied by just a few authors. A contribution in this direction is [12] where the authors use a topological method to study the case $N=1$. Another contribution was made by João Marcos B. do Ó in [14] who studied the case when $F(x, s)$ interacts only with the first eigenvalue. In this paper, we will consider three situations.

The first situation is the resonance on the right side of the first eigenvalue, we will prove the following results :

Theorem 1.1. Suppose that

$\left.c_{1.1}\right) \quad \lim _{|s| \rightarrow+\infty}[s f(x, s)-p F(x, s)]=-\infty \quad$ uniformly for a.e. $x \in \Omega$

$\left.c_{2.1}\right) \quad \lambda_{1} \leq \liminf _{|s| \rightarrow+\infty} \frac{p F(x, s)}{|s|^{p}}$ uniformly for a.e. $x \in \Omega$ 
$\left.c_{3.1}\right) \quad \limsup _{s \rightarrow 0} \frac{p F(x, s)}{|s|^{p}} \leq \beta<\lambda_{1} \quad$ uniformly for a.e. $x \in \Omega$ then the problem (1.1) has a nontrivial solution $u \in W_{0}^{1, p}(\Omega)$.

Theorem 1.2. Suppose that

$\left.c_{1.2}\right) \quad \lim _{|s| \rightarrow+\infty}[s f(x, s)-p F(x, s)]=+\infty \quad$ uniformly for a.e. $x \in \Omega$

$\left.c_{2.2}\right) \quad F(x, s) \leq A|s|^{p}+B(x) \quad B(.) \in L^{1}(\Omega)$

$\left.c_{3.2}\right) \quad \limsup _{s \rightarrow 0} \frac{p F(x, s)}{|s|^{p}} \leq \beta<\lambda_{1} \quad$ uniformly for a.e. $x \in \Omega$

$\left.c_{4.2}\right) \quad \int_{\Omega} F\left(x, t_{0} \varphi_{1}\right) d x-\frac{t_{0}^{p}}{p}>0$ for at least one $t_{0}>0$.

$\varphi_{1}$ is a $\lambda_{1}$-eigenfunction with $\left\|\varphi_{1}\right\|=\left(\int_{\Omega}\left|\nabla \varphi_{1}\right|^{p}\right)^{\frac{1}{p}}=1$. Then the problem (1.1) possesses a nonzero solution $u \in W_{0}^{1, p}(\Omega)$.

\section{Remarks}

1. the condition $c_{3.1}$ ) and $c_{3.2}$ ) can be replaced by

$$
F(x, s) \leq 0 \text { for }|s| \leq \delta \quad(\delta>0)
$$

2. In the condition $c_{1.2}$ ) of theorem 1.2 we can replace $+\infty$ by $-\infty$, in this case the theorem can be proved without condition $c_{2.2}$ ).

The second situation is the resonance between the two first consecutive eigenvalues. To state our result, let us denote by $l(x), k(x)$ and $\tilde{L}(x)$ the corresponding limits $l(x)=\liminf _{|s| \rightarrow+\infty} \frac{f(x, s)}{|s|^{p-2} s} ; k(x)=\limsup _{|s| \rightarrow+\infty} \frac{f(x, s)}{|s|^{p-2} s} ; \tilde{L}(x)=\liminf _{|s| \rightarrow+\infty}[p F(x, s)-$ $s f(x, s)]$.

These limits are taken uniformly for a.e. $x \in \Omega$.

Theorem 1.3. Suppose that

$\left.c_{1.3}\right) \quad \lambda_{1} \leq l(x) \leq k(x) \leq \beta<\lambda_{2}$

$\left.c_{2.3}\right) \quad \tilde{L}(.) \in L^{1}(\Omega)$ and $\int_{\Omega} \tilde{L}(x) d x \geq 0$

$\left.c_{3.3}\right) \quad F(x, s) \leq 0$ for $|s| \stackrel{\Omega}{\leq} \delta$ with $(\delta>0)$

$\left.c_{4.3}\right) \quad$ there exists $t_{0}>0$ such that $\int_{\Omega} F\left(x, t_{0} \varphi_{1}(x)\right) d x-\frac{t_{0}^{p}}{p}>0$.

Then the problem (1.1) has a nontrivial solution. 


\section{Remarks}

1. Instead of the condition $c_{2.3}$ ), we can assume

$$
\limsup _{|s| \rightarrow+\infty}[p F(x, s)-s f(x, s)]=\tilde{K}(.) \in L^{1}(\Omega) \text { and } \int_{\Omega} \tilde{K}(x) d x \leq 0
$$

2. the condition $c_{3.3}$ ) can be replaced by

$$
\limsup _{s \rightarrow 0} \frac{p F(x, s)}{|s|^{p}} \leq \beta<\lambda_{1}
$$

The third situation is the resonance on the left side of the first eigenvalue, we will prove the following :

Theorem 1.4. Assume that

$\left.c_{1.4}\right) \quad|F(x, s)| \leq A|s|^{p}+B$

$\left.c_{2.4}\right) \quad K(x)=\limsup _{|s| \rightarrow+\infty} \frac{p F(x, s)}{|s|^{p}} \leq \lambda_{1} \quad$ uniformly for a.e. $x \in \Omega$.

$\left.c_{3.4}\right)$ there exists $R(.) \in L^{1}(\Omega)$ such that $\int_{\Omega} R(x) d x \geq 0$ and

$$
\liminf _{|s| \rightarrow+\infty}[p F(x, s)-s f(x, s)] \geq R(x) \quad \text { uniformly for a.e. } x \in \Omega
$$

$\left.c_{4.4}\right) \quad F(x, s) \leq 0$ for $|s| \leq \delta(\delta>0)$

$\left.c_{5.4}\right) \quad$ there exists $t_{0}>0$ such that $\int_{\Omega} F\left(x, t_{0} \varphi_{1}(x)\right) d x-\frac{t_{0}^{p}}{p}>0$

Then the problem (1.1) possesses a nonzero solution.

In the final section, we will give examples to illustrate our results.

\section{PROOF OF THE MAIN RESULTS}

We start recalling a compactness condition of the Palais Smale type which was introduced by Cerami and which allows rather general minimax results.

A functional $I \in^{1}(E),$,$E is a real Banach space, is said to satisfy the$ condition () at the level $c\left(()_{c}\right)$ if the following holds : 
$\left.c_{i}\right)$ any bounded sequence $\left(u_{n}\right)$ such that $I\left(u_{n}\right) \rightarrow c$ and $I^{\prime}\left(u_{n}\right) \rightarrow 0$ possesses a convergent subsequence.

$\left.c_{i i}\right)$ there exists constants $\delta, R, \alpha>0$ such that $\left\|I^{\prime}(u)\right\|\|u\| \geq \alpha$ for any $u \in I^{-1}([c-\delta, c+\delta])$ with $\|u\| \geq R$.

Remark. Using assumption $\left(f_{0}\right)$ the functional

$$
\Phi(u)=\int_{\Omega} \frac{1}{p}|\nabla u|^{p}-\int_{\Omega} F(x, u(x)) d x
$$

is well defined and of class ${ }^{1}$ on the Sobolev space $W_{0}^{1, p}(\Omega)$ with derivative

$$
\Phi^{\prime}(u) v=\int_{\Omega}|\nabla u|^{p-2} \nabla u \nabla v-\int_{\Omega} f(x, u) v d x \quad \text { for all } u, v \in W_{0}^{1, p}(\Omega) .
$$

Thus, the critical points of $\Phi$ are precisely the weak solutions of (1.1). Moreover, the condition $c_{i}$ ) yields for every $c \in$.

Denote the norm in $W_{0}^{1, p}(\Omega)$ by $\|\| \quad.\left(\|u\|^{p}=\int_{\Omega}|\nabla u|^{p}\right)$ and the norm in $L^{q}(\Omega)$ by $\|\cdot\|_{q}\left(\|u\|_{q}=\left(\int_{\Omega}|u|^{q}\right)^{\frac{1}{q}}\right)$. To obtain a nontrivial critical point of the functional $\Phi$, we will apply the following version of the Mountain-Pass theorem, with condition ()

Theorem 2.1. Let $E$ be a real Banach space and $I \in^{1}(E$, ) satisfying condition ()$_{c}$, for every $c>0$.

Suppose that $I(0)=0$, and for some $\alpha, \rho>0$ and $e \in E$ with $\|e\|>\rho$, one has $\alpha \leq \inf _{\|u\|=\rho} I(u)$ and $I(e)<0$, then $I$ has a critical value $c \geq \alpha$ characterized by

$c=\inf _{h \in \Gamma} \sup _{0 \leq t \leq 1} I(h(t))$ where $\Gamma=h \in([0,1], E): h(0)=0, h(1)=e$.

Remark. It is not difficult to see that the same proof of the standard Mountain-Pass theorem applies to the present context, since the deformation theorem, (theorem 1.3) in [5] is obtained with condition () in Banach space.

To prove the theorems in the first situation, we need the following preliminary lemmas. 
Lemma 2.1. Assume $\left(f_{0}\right)$ and $\left.c_{1.1}\right)$ if $c_{3.1}$ ) holds then, there exists $\rho, \alpha>0$ such that

$$
\Phi(u) \geq \alpha \quad \text { if } \quad\|u\|=\rho
$$

Proof. Using $\left(f_{0}\right)$ and $\left.c_{1.1}\right)$ it is easy to show that

$$
F(x, s) \leq A|s|^{p}+B
$$

for some constants $A, B>0$.

Choosing $\varepsilon>0$ such that $\beta+\varepsilon<\lambda_{1}$, in view of $c_{3.1}$ ) and the inequality (1) there exists $\tilde{A}=\tilde{A}(\varepsilon) \geq 0$ such that

$$
F(x, s) \leq \frac{1}{p}(\beta+\varepsilon)|s|^{p}+\tilde{A}|s|^{q}
$$

we may assume $q>p$, with the Poincaré inequality $\lambda_{1}\|u\|_{p}^{p} \leq\|u\|^{p}$ and the Sobolev inequality $\|u\|_{q}^{q} \leq K\|u\|^{q}$, we obtain the estimate

$$
\Phi(u) \geq \frac{1}{p}\left(1-\frac{\beta+\varepsilon}{\lambda_{1}}\right)\|u\|^{p}-\tilde{A} K\|u\|^{q} .
$$

Thus

$$
\Phi(u) \geq\left(\frac{1}{p}\left(1-\frac{\beta+\varepsilon}{\lambda_{1}}\right)-\tilde{A} K\|u\|^{q-p}\right)\|u\|^{p} .
$$

So taking $\rho=\left[\frac{1}{2 p}\left[1-\frac{\beta+\varepsilon}{\lambda_{1}}\right] \frac{1}{\tilde{A} K}\right]^{\frac{1}{q-p}}$ and $\alpha=\frac{1}{2 p}\left(1-\frac{\beta+\varepsilon}{\lambda_{1}}\right) \rho^{p}$, we obtain $\Phi(u) \geq \alpha$ if $\|u\|=\rho$. Then the proof of lemma 2.1 is now complete.

The next result is standard (cf [7] e.g )

Lemma 2.2. Assume $c_{1.1}$ ) and $c_{2.1}$ ), then we have

$$
\lim _{|s| \rightarrow+\infty} F(x, s)-\frac{\lambda_{1}}{p}|s|^{p}=+\infty \quad \text { uniformly for a.e. } x \in \Omega
$$

It follows from lemma 2.2 above that there exists $R_{0}>0$ such that

$$
F(x, s)-\frac{\lambda_{1}}{p}|s|^{p} \geq 0 \quad \text { for all }|s| \geq R_{0} .
$$


On the other hand we claim $\left(F\left(x, t \varphi_{1}(x)\right)-\frac{\lambda_{1}}{p}\left|t \varphi_{1}\right|^{p}\right)_{t \in}$ is bounded below ( $\varphi_{1}$ is $\lambda_{1}$ normalized eigenfunction: that is $\left\|\varphi_{1}\right\|^{p}=\lambda_{1} \int_{\Omega}\left|\varphi_{1}\right|^{p}=$ 1 ), Indeed, we consider the set $\Omega_{0}=\left\{x \in \Omega:\left|t \varphi_{1}(x)\right| \geq R_{0}\right\}$, in view of (2) we have

$$
F\left(x, t \varphi_{1}(x)\right)-\frac{\lambda_{1}}{p}\left|t \varphi_{1}\right|^{p} \geq 0 \quad \text { for all } x \in \Omega_{0} .
$$

If $x \notin \Omega_{0},\left(f_{0}\right)$ yields

$$
F\left(x, t \varphi_{1}(x)\right)-\frac{\lambda_{1}}{p}\left|t \varphi_{1}\right|^{p} \geq B_{0} \quad \text { for some } B_{0} \in
$$

and using (3) and (4) the desired result follows.

Lemma 2.3. Assume $\left.\left(f_{0}\right), c_{1.1}\right)$ and $\left.c_{2.1}\right)$, then there exists $R_{1}>0\left(R_{1}>\right.$ $\rho)$ such that

$$
\int_{\Omega} F\left(x, R_{1} \varphi_{1}(x)\right) d x-\frac{R_{1}^{p}}{p}>0 .
$$

This means $\Phi\left(R_{1} \varphi_{1}\right)<0$.

Proof. Suppose by negation that there exists a sequence $\left(t_{n}\right)$ such that

$$
\left|t_{n}\right| \rightarrow+\infty \text { and } \int_{\Omega} F\left(x, t_{n} \varphi_{1}(x)\right) d x-\frac{\left|t_{n}\right|^{p}}{p} \leq 0
$$

Since $\lambda_{1} \int_{\Omega}\left|\varphi_{1}\right|^{p} d x=1,(5)$ is equivalent to

$$
\int_{\Omega}\left(F\left(x, t_{n} \varphi_{1}(x)\right)-\frac{\lambda_{1}}{p}\left|t_{n} \varphi_{1}\right|^{p}\right) d x \leq 0
$$

thus

$$
\liminf _{n \rightarrow+\infty} \int_{\Omega}\left(F\left(x, t_{n} \varphi_{1}(x)\right)-\frac{\lambda_{1}}{p}\left|t_{n} \varphi_{1}\right|^{p}\right) d x \leq 0 .
$$

On the other hand, using lemma 2.2, Fatou's lemma with 


$$
\begin{aligned}
& h_{n}=F\left(x, t_{n} \varphi_{1}(x)\right)-\frac{\lambda_{1}}{p}\left|t_{n} \varphi_{1}\right|^{p} \text { gives } \\
& \quad \liminf _{n \rightarrow+\infty} \int_{\Omega} h_{n}(x) d x \geq \int_{\Omega} \liminf _{n \rightarrow+\infty} h_{n}(x) d x=+\infty
\end{aligned}
$$

which contradicts (6), then the proof is complete.

Lemma 2.4. Assume $\left(f_{0}\right)$ and $\left.c_{1.1}\right)$, then the functional $\Phi$ satisfies ()$_{c}$ for every $c \in$.

Proof. Let us assume by negation, that $\Phi$ does not satisfy () $c$ for some $c \in$, then there exists a sequence $\left(u_{n}\right)$ such that

$$
\Phi^{\prime}\left(u_{n}\right) u_{n} \rightarrow 0, \quad \Phi\left(u_{n}\right) \rightarrow c, \text { and }\left\|u_{n}\right\| \rightarrow+\infty
$$

It follows that

$$
\lim _{n \rightarrow+\infty} \int_{\Omega}\left(p F\left(x, u_{n}\right)-u_{n} f\left(x, u_{n}\right)\right) d x=-p c .
$$

A subsequence of $v_{n}\left(v_{n}=\frac{u_{n}}{\left\|u_{n}\right\|}\right)$ (still denoted by $\left.\left(v_{n}\right)\right)$ is such that

$$
\begin{array}{ccc}
v_{n} \rightarrow v & \text { weakly in } W_{0}^{1, p}(\Omega) \\
v_{n} \rightarrow v & \text { strongly in } L^{p}(\Omega) \\
v_{n}(x) \rightarrow v(x), \text { a.e. } x \in \Omega & \text { and }\left|v_{n}(x)\right| \leq z(x), z(.) \in L^{p}(\Omega) .
\end{array}
$$

Using (1) and (7) we conclude that

$$
\frac{1}{p}\left\|u_{n}\right\|^{p}-A\left\|u_{n}\right\|_{p}^{p}-B \leq K^{\prime}
$$

for some constant $K^{\prime}$, therefore

$$
\frac{1}{p}-A\|v\|_{p}^{p} \leq 0
$$

So that $v \neq 0$. Let us define $\Omega_{1}=\{x \in \Omega: v(x) \neq 0\}$, we have

$$
\text { mes }\left(\Omega_{1}\right)>0 \text { and }\left|u_{n}(x)\right| \rightarrow+\infty \text { a.e. } x \in \Omega_{1}
$$


using $\left(f_{0}\right)$ and $\left.c_{1.1}\right)$, we conclude that

$$
p F\left(x, u_{n}\right)-u_{n} f\left(x, u_{n}\right) \geq M \quad \text { for some constant } M \in
$$

and

$$
\lim _{n \rightarrow+\infty}\left(p F\left(x, u_{n}\right)-u_{n} f\left(x, u_{n}\right)\right)=+\infty \quad \text { a.e. } x \in \Omega_{1}
$$

However, Fatou's lemma gives

$$
\lim _{n \rightarrow+\infty} \int_{\Omega}\left(p F\left(x, u_{n}\right)-u_{n} f\left(x, u_{n}\right)\right) d x=+\infty
$$

which contradicts (8) and shows that (7) can not occur. Then the proof of lemma 2.4 is complete.

Proof of theorem 1.1. In view of lemmas 2.1, 2.3, 2.4 we may apply theorem 2.1 taking $e=R_{1} \varphi_{1}$, it follows that the functional $\Phi$ has a critical value $c_{0} \geq \alpha>0$, and, hence that problem (1.1) has a nontrivial solution $u_{0} \in W_{0}^{1, p}(\Omega)$.

The proof of theorem 1.2. is similar to that of theorem 1.1 and is omitted. To prove the theorem 1.3 we will use the following lemmas.

Lemma 2.5. Assume $\left.\left(f_{0}\right), c_{1.3}\right)$ and $\left.c_{2.3}\right)$ then the functional $\Phi$ satisfies $(C) c$ for every $c>0$.

Proof. From $\left(f_{0}\right)$ and $c_{1.3}$ it follows that there exists constants $a$ and $b$ such that

$$
|f(x, s)| \leq a|s|^{p-1}+b .
$$

Now, suppose by negation, that $\Phi$ does not satisfy () $c$ for some $c>0$, then there exists a sequence $\left(u_{n}\right)$ such that $(7)$ holds.

Let us define $v_{n}=\frac{u_{n}}{\left\|u_{n}\right\|}, f_{n}=\frac{f\left(x, u_{n}\right)}{\left\|u_{n}\right\|^{p-1}}$, passing to subsequence of $v_{n}$ (respectively $f_{n}$ ), still denoted by $\left(v_{n}\right)$ (respectively $f_{n}$ ) we may assume that :

$v_{n} \rightarrow v$ weakly in $W_{0}^{1, p}(\Omega), v_{n} \rightarrow v$ strongly in $L^{p}(\Omega)$ and a.e. $x \in \Omega$, $f_{n} \rightarrow \tilde{f}$ in $L^{p}(\Omega)$.

We have the following claim which is inspired from [7].

\section{Claim 1}


1. $\lambda_{1} \leq \frac{\tilde{f}}{|v|^{p-2} v} \leq \beta \quad$ if $\quad v \neq 0$

2. $\tilde{f}(x)=0$ if $\quad v=0$.

0 .

Letting, $m()=.\frac{\tilde{f}}{|v|^{p-2} v} \quad$ if $v \neq 0 \quad$ and $\quad m()=.\frac{1}{2}\left(\lambda_{1}+\beta\right) \quad$ if $v=$ By (7) we have $\left|\Phi^{\prime}\left(u_{n}\right) w\right| \leq \varepsilon_{n}\|w\|$ for all $w \in W_{0}^{1, p}(\Omega)$, where $\varepsilon_{n} \rightarrow 0$, therefore

$$
\frac{\left|\Phi^{\prime}\left(u_{n}\right) u_{n}\right|}{\left\|u_{n}\right\|^{p}}=\left|1-\int_{\Omega} \frac{f\left(x, u_{n}\right.}{\left\|u_{n}\right\|^{p-1}} v_{n}\right| \leq \frac{\varepsilon_{n}}{\left\|u_{n}\right\|^{p-1}}
$$

hence

$$
\int_{\Omega} \frac{f\left(x, u_{n}\right)}{\left\|u_{n}\right\|^{p-1}} v_{n} \rightarrow 1
$$

passing to the limit, we obtain $\int_{\Omega} \tilde{f} v=1$, so that $v \neq 0$. On the other hand, for any $w \in W_{0}^{1, p}(\Omega)$ we have

$$
\left|\frac{\Phi^{\prime}\left(u_{n}\right)}{\| u_{n}||^{p-1}} w\right|=\left.\left|\int_{\Omega}\right| \nabla v\right|^{p-2} \nabla v \nabla w-\int_{\Omega} \frac{f\left(x, u_{n}\right)}{\left\|u_{n}\right\|^{p-1}} w \mid \leq \varepsilon_{n} \frac{\|w\|}{\left\|u_{n}\right\|^{p-1}}
$$

passing to the limit, we conclude

$$
\int_{\Omega}|\nabla v|^{p-2} \nabla v \nabla w-\int_{\Omega} \tilde{f} w=0
$$

that is

$$
\int_{\Omega}|\nabla v|^{p-2} \nabla v \nabla w-\int_{\Omega} m(.)|v|^{p-2} v w=0 \quad \forall w \in W_{0}^{1, p}
$$

in other words, $v$ is a weak solution of the following problem

$$
\left(P_{m}\right)\left\{\begin{array}{cccc}
-\Delta_{p} u & = & m(.)|u|^{p-2} u & \text { in } \Omega \\
u & = & 0 & \text { on } \partial \Omega
\end{array}\right.
$$

The result above and claim 1 imply

$$
1 \in \sigma\left(-\Delta_{p}, m(.)\right) \text { and } \lambda_{1} \leq m(.) \leq \beta<\lambda_{2}
$$


if $\lambda_{1} m($.$) (that is \lambda_{1}<m($.$) on subset of \Omega$ of positive measure), then by the second part of (10), the strict monotonicity of $\lambda_{1}$ (cf [11]) and the strict partial monotonicity of $\lambda_{2}$ (cf [4]), we have

$$
\lambda_{1}(m(.))<\lambda_{1}\left(\lambda_{1}(1)\right)=1 \text { and } \lambda_{2}(m(.))>\lambda_{2}\left(\lambda_{2}(1)\right)=1
$$

thus

$$
\lambda_{1}(m(.))<1<\lambda_{2}(m(.)) .
$$

Since $\left.\sigma\left(-\Delta_{p}, m().\right) \cap\right] \lambda_{1}(m()),. \lambda_{2}(m()).[=\emptyset \quad(\mathrm{cf}[4])$, the first part of (10) and (11) are in contradiction, hence $m()=.\lambda_{1}$ and $v$ is a $\lambda_{1}$ eigenfunction, so it follows that

$$
\left|u_{n}(x)\right| \rightarrow+\infty \text { a.e. } x \in \Omega .
$$

On the other hand by (8) we have

$$
\lim _{n \rightarrow+\infty} \int_{\Omega} p F\left(x, u_{n}\right)-u_{n} f\left(x, u_{n}\right) d x=-p c
$$

combining (12) and $c_{2.3}$ ), Fatou's lemma yields

$$
\int_{\Omega} \tilde{L}(x) d x \leq \liminf _{n \rightarrow+\infty} \int_{\Omega} p F\left(x, u_{n}\right)-u_{n} f\left(x, u_{n}\right) d x .
$$

Via (13) we obtain

$$
\int_{\Omega} \tilde{L}(x) d x \leq-p c<0
$$

which gives a contradiction, then the proof of lemma 2.5 is complete.

Lemma 2.6. Assume $\left.\left(f_{0}\right), c_{1.3}\right)$ and $\left.c_{3.3}\right)$, then there exists $\rho, \alpha>0$ such that $\Phi(u) \geq \alpha$ if $\|u\|=\rho$

Proof. Since $\int_{|u(x)| \leq \delta} F(x, u(x)) d x \leq 0$, we have

$$
\Phi(u) \geq \frac{1}{p}\|u\|^{p}-\int_{|u(x)|>\delta} F(x, u(x)) d x .
$$

On the other hand we have

$$
\limsup _{\|u\| \rightarrow 0} \int_{|u(x)|>\delta} \frac{F(x, u(x))}{\|u\|^{p}} d x \leq 0
$$


indeed, assume that (14) is false, then we can find a sequence $\left(u_{n}\right)$ and $\varepsilon>0$ such that

$$
\left\|u_{n}\right\| \rightarrow 0 \quad \text { and } \quad \int_{|u(x)|>\delta} \frac{F\left(x, u_{n}(x)\right)}{\left\|u_{n}\right\|^{p}} \geq \varepsilon .
$$

From (9) we deduce

$$
F(x, s) \leq \tilde{K}|s|^{p} \quad \text { for }|s| \geq \delta,
$$

and by (15) and (16), we conclude

$$
\int_{\Omega} \tilde{K}\left|v_{n}(x)\right|^{p} \chi_{n}(x) d x \geq \varepsilon,
$$

where $v_{n}=\frac{u_{n}}{\left\|u_{n}\right\|}, \chi_{n}(x)=1$ if $\left|u_{n}(x)\right|>\delta$ and $\chi_{n}=0$ if $\left|u_{n}(x)\right| \leq \delta$. Since $\left\|u_{n}\right\| \rightarrow 0$, then $u_{n}(x) \rightarrow 0$ and $\chi_{n} \rightarrow 0$, so passing to the limit in the inequality (17) we get a contradiction.

Now, via (14) choosing $\rho>0\left(\rho<t_{0}, t_{0}\right.$ given in $\left.\left.c_{4.3}\right)\right)$ such that

$$
\int_{|u(x)|>\delta} \frac{F(x, u(x))}{\|u\|^{p}} \leq \frac{1}{2 p} \text { for }\|u\|=\rho,
$$

then, for $\|u\|=\rho$ we obtain

$$
\Phi(u) \geq \frac{1}{2 p} \rho^{p} .
$$

To conclude the proof, take $\alpha=\frac{1}{2 p} \rho^{p}$.

Proof of theorem 1.3. In view of lemmas 2.5 and 2.6 we may apply theorem 2.1 letting $e=t_{0} \varphi_{1}$. It follows that the functional $\Phi$ has a critical value $c \geq \alpha>0$.

Lemma 2.7. Assume $\left.\left.\left.\left(f_{0}\right), c_{1.4}\right), c_{2.4}\right), c_{3.4}\right)$ then the functional $\Phi$ satisfies the condition () $c$ for every $c>0$.

Proof. Assume by contradiction that there exists $c>0$ and a sequence $\left(u_{n}\right)$ in $W_{0}^{1, p}(\Omega)$ such that $(7)$ holds. Then a subsequence of $\left(v_{n}\right)$, still denoted by $\left(v_{n}\right)$, where $\left(v_{n}=\frac{u_{n}}{\left\|u_{n}\right\|}\right)$ is such that

$$
v_{n} \rightarrow v \quad \text { weakly in } W_{0}^{1, p}(\Omega)
$$




$$
\begin{gathered}
v_{n} \rightarrow v \quad \text { strongly in } L^{p}(\Omega) \\
v_{n}(x) \rightarrow v(x) \text {, a.e. } x \in \Omega \\
\left|v_{n}(x)\right| \leq h(x) \text { where } h(.) \in L^{p} .
\end{gathered}
$$

In view of (7) we have

$$
\frac{1}{p}\left\|u_{n}\right\|^{p}-\int_{\Omega} F\left(x, u_{n}\right) d x \leq c^{\prime} \quad\left(c^{\prime} \in\right)
$$

thus by $c_{1.4}$ ) and $c_{2.4}$ we obtain

$$
\frac{1}{p}\left\|u_{n}\right\|^{p}-\frac{\left(\lambda_{1}+\varepsilon\right)}{p}\left\|u_{n}\right\|_{p}^{p}-\|B\| \leq c^{\prime}
$$

then

$$
\frac{1}{p}-\frac{\left(\lambda_{1}+\varepsilon\right)}{p}\left\|v_{n}\right\|_{p}^{p}-0(n) \leq 0(n) .
$$

Passing to the limit in the above inequality, we obtain

$$
\frac{1}{p}-\frac{\lambda_{1}}{p}\|v\|_{p}^{p} \leq 0 .(18)
$$

Since $\lambda_{1}\|v\|_{p}^{p} \leq\|v\|^{p} \leq 1$, from (18) we conclude that

$$
v \neq 0 \text { and }\|v\|^{p}=\lambda_{1}\|v\|_{p}^{p}
$$

hence $v$ is a $\lambda_{1}$ eigenfunction, therefore

$$
\left|u_{n}(x)\right| \rightarrow+\infty \quad \text { a.e. } x \in \Omega .
$$

On the other hand (7) gives

$$
\lim _{n \rightarrow+\infty} \int_{\Omega}\left(p F\left(x, u_{n}\right)-u_{n} f\left(x, u_{n}\right)\right) d x=-p c .
$$

However, by (19), Fatou's lemma gives

$$
\int_{\Omega} R(x) d x \leq-p c<0
$$

which contradicts $c_{3.4}$ ). Then the functional $\Phi$ satisfies ()$c$ for every $c>0$.

Proof of theorem 1.4. Combining lemmas 2.6, 2.8 and taking $e=$ $t_{0} \varphi_{1}\left(t_{0}>\rho\right)$ in theorem2.1 to conclude the existence of a critical point $u_{0} \in W_{0}^{1, p}(\Omega)$ of $\Phi$ with $\Phi\left(u_{0}\right) \geq \alpha>0$. 


\section{SOME EXAMPLES}

This final section treats the question of verifying some applications of the hypotheses that are required in the abstract theorems presented earlier.

\section{Example 1}

We consider the boundary value problem

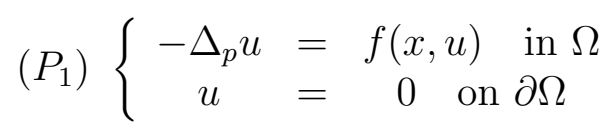

where :

$$
f(x, s)=\left\{\begin{array}{cc}
\lambda_{1} s^{p-1}+\frac{\lambda_{1}}{p s} & \text { if } \quad s \geq 1 \\
\lambda_{1}\left(\frac{p+1}{p}\right) s^{p} & \text { if } \quad 1 \geq s \geq 0 \\
-f(x,-s) & \text { if } \quad s \leq 0 .
\end{array}\right.
$$

The primitive $F(x, s)=\int_{0}^{s} f(x, t) d t$ is such that

$$
F(x, s)=\left\{\begin{array}{cc}
\lambda_{1} \frac{s^{p}}{p}+\lambda_{1} \frac{\log (s)}{p} & \text { if } \quad s \geq 1 \\
\lambda_{1} \frac{s^{p+1}}{p} & \text { if } \quad 1 \geq s \geq 0 \\
F(x,-s) & \text { if } \quad 0 \geq s .
\end{array}\right.
$$

A simple computation shows that:

1. $\lim _{|s| \rightarrow+\infty} \frac{p F(x, s)}{|s|^{p}}=\lambda_{1}$

2. $\lim _{s \rightarrow 0} \frac{p F(x, s)}{|s|^{p}}=0$

3. $\lim _{|s| \rightarrow+\infty}[s f(x, s)-p F(x, s)]=-\infty$.

Hence the hypotheses of the theorem 1.1 are satisfied, and $\left(P_{1}\right)$ is a resonant problem.

Example 2

We shall now construct as above a Carathéodory function $f$ satisfying 
all conditions of theorem 1.3 , and such that :

$$
\liminf _{|s| \rightarrow+\infty} \frac{f(x, s)}{|s|^{p-2} s}=\lambda_{1}
$$

and

$$
\limsup _{|s| \rightarrow+\infty} \frac{f(x, s)}{|s|^{p-2} s}=\beta
$$

where $\lambda_{1}<\beta<\lambda_{2}$, so taking

$$
f(x, s)=\left\{\begin{array}{cc}
\lambda_{1} s^{p-1}+\frac{p \lambda_{1}}{s^{2}} & \text { if } \quad s \geq 1 \\
(p+1) \lambda_{1} s^{p} & \text { if } \quad 1 \geq s \geq 0 \\
-2 \beta|s|^{p} \quad \text { if } \quad 0 \geq s \geq-1 \\
\beta|s|^{p-2} s-\frac{\beta}{s^{2}} \quad \text { if } \quad-1 \geq s .
\end{array}\right.
$$

A simple calculation shows that the primitive $F$ satisfies :

$$
\begin{aligned}
& \liminf _{|s| \rightarrow+\infty} \frac{p F(x, s)}{|s|^{p}}=\lambda_{1} \\
& \limsup _{|s| \rightarrow+\infty} \frac{p F(x, s)}{|s|^{p}}=\beta
\end{aligned}
$$

and

$$
\limsup _{s \rightarrow 0} \frac{p F(x, s)}{|s|^{p}}=0 .
$$

$\liminf _{|s| \rightarrow+\infty}(p F(x, s)-s f(x, s)) \geq \inf \left(\lambda_{1}\left(p^{2}+p-1\right), \beta\left(p-1+\frac{2 p}{p+1}\right)\right)>0$.

Hence by theorem 1.3 the problem $\left(P_{1}\right)$ possesses a nonzero solution $u \in W_{0}^{1, p}(\Omega)$.

\section{Example 3}

In this example we consider the Dirichlet problem $\left(P_{1}\right)$ where the 
Carathéodory function $f$ is as follows :

$$
f(x, s)=\left\{\begin{array}{cc}
\lambda_{1} s^{p-1}+\frac{\lambda_{1}}{p s^{2}}+p \frac{\lambda_{1}}{s^{2}} & \text { if } \quad s \geq 1 \\
\frac{\lambda_{1}}{p}[p(p+1)+1] s^{p(p+1)} & \text { if } \quad 1 \geq s \geq 0 \\
-f(x,-s) & \text { if } \quad 0 \geq s .
\end{array}\right.
$$

The primitive $F(x, s)=\int_{0}^{s} f(x, t) d t$ is such that

$$
F(x, s)=\left\{\begin{array}{cc}
\frac{\lambda_{1} s^{p}}{p}-\frac{\lambda_{1}}{p s}+\frac{\lambda_{1}}{p}-p \frac{\lambda_{1}}{s}+p \lambda_{1} & \text { if } \quad s \geq 1 \\
\frac{\lambda_{1}}{p} s^{p(p+1)+1} & \text { if } \quad 1 \geq s \geq 0 \\
F(x,-s) & \text { if } \quad 0 \geq s .
\end{array}\right.
$$

A simple computation shows that

1. $\lim _{|s| \rightarrow+\infty} \frac{p F(x, s)}{|s|^{p}}=\lambda_{1}$

2. $\lim _{s \rightarrow 0} \frac{p F(x, s)}{|s|^{p}}=0$

3. $\lim _{|s| \rightarrow+\infty}\left[p F(x, s)-\frac{\lambda_{1} s^{p}}{p}\right]=\lambda_{1}\left(p+\frac{1}{p}\right)$

4. $\liminf \operatorname{ins}_{|s|+\infty}[p F(x, s)-s f(x, s)]=\lambda_{1}+p^{2} \lambda_{1}$.

Hence by theorem 1.4 the problem $\left(P_{1}\right)$ possesses a nonzero solution in $W_{0}^{1, p}(\Omega)$. 


\section{References}

[1] R.A. Adams, Sobolev spaces. Academic Press, New York, (1975).

[2] A. Anane Simplicité et isolation de la première valeur propre du p-laplacien avec poids. C. R. Ac. Sc. Paris, 305, pp. $725-728$, (1987).

[3] A. Anane, J. P. Gossez Strongly nonlinear elliptic problems near resonance: A variational approach. Comm. Part. Diff. Eq. 15(8), pp. 1141-1159, (1990).

[4] A. Anane, N. Tsouli On the second eigenvalue of the p-laplacian, Nonlinear Partial Differential Equations, Pitman Research Notes 343, pp. 1-9, (1996).

[5] Bartolo P., Benci and Fortunato D. Abstrat critical point theorems and applications to some nonlinear problems with strong resonance at infinity, Nonlinear Analysis 7, pp. 981-1012, (1983).

[6] D. G. Costa, A. S. Oliveira Existence of solution for a class of semilinear elliptic problems at double resonance. Bol. Soc. BRAS. Mat., vol 19, pp. 21-37, (1988).

[7] D.G. COSTA, C.A. Magalhçes Variational elliptic problems which are nonquadratic at infinity. Nonlinear Analysis, vol 23. No 11, pp. 1401-1412, (1994).

[8] M. Cuesta, D. de Figueiredo, J. P. Gossez The beginning of the Fucik spectrum for the p-laplacian. J. Diff. Equat., 159 , pp. 212238, (1999).

[9] D. G. de Figueiredo, J. P. Gossez, Conditions de non résonance pour certains problèmes elliptiques semi-linéaire, C. R. Acad. Sci. Paris 302, pp. 543-545, (1986).

[10] D. G. de Figueireido, J. P. Gossez, Nonresonance below the first eigenvalue for a semilinear elliptic problems. Math. An. 281, pp. 589-610 (1988). 
[11] D. G. de Figueiredo, J.P. Gossez, Strict monotonicity of eigenvalues and unique contination. Comm. Part. Diff. Eq., 17, pp. 339-346, (1992).

[12] A. R. EL Amrouss, M. Moussaoui Non resonance entre les deux premières valeurs propres d'un problème quasi-lineaire. Bul. Bel. Math. Soc. 4, (1997).

[13] J. Mawhin, J.R. Ward, M. Willem, Variational methods of semilinear elliptic equations. Arch. Rat. Mech. An 95, pp. 269-277, (1986).

[14] João. Marcos. B. do Ó. Solution to perturbed eigenvalue problems of the p-laplacian in ${ }^{N *}$. J. D. E. N 11, pp. 1-15 (1997).

[15] M. Moussaoui, J.P. Gossez, A note on noresonance between consecutive eigenvalues for a semilinear elliptic problem Pitman Res. Notes in Math., 343, pp. 155 - 166, (196).

[16] P.H. Rabinowitz, Some minimax theorems and applications to nonlinear partial diffirential equations, Nonlinear Analysis, Cesari, Kannan and Weinberger. Eds, 161-177, Academic Press, Orlando, Fl. (1978).

[17] P.H. Rabinowitz, Some minimax methods in critical point theory with application to differential equations, CBMS, Regional conf. Ser. Math., vol 65 AMS, Providence Ri. (1986).

[18] M Schechter, Nonlinear elliptic boundary value problems at strong resonance, Amer. J. Math., 112, pp. 439-460, (1990).

[19] E. A. B. Silva, Linking theorems and applications to semilinear elliptic problems at resonance, Nonlinear Analysis TMA, 16, pp. 455-477, (1991).

Received : July, 2000. 


\section{Moussaoui and M. Moussaoui}

Department of Mathematics

Faculty of Sciences

University Mohamed I

P. O. Box 524

60000 Oujda

Morocco

E-MAIL : moussaoui@sciences.univ-oujda.ac.ma 